Original Research Paper

\title{
ANFIS Aided AODV Routing Protocolfor Mobile Ad Hoc Networks
}

\author{
Vivek Sharma, Bashir Alam and M.N. Doja \\ Department of Computer Engineering, Jamia MilliaIslamia New Delhi, 110025, India
}

\author{
Article history \\ Received: 16-08-2017 \\ Revised: 31-08-2017 \\ Accepted: 6-10-2017 \\ Corresponding author: \\ Vivek Sharma \\ Department of Computer \\ Engineering, Jamia \\ MilliaIslamia New Delhi, \\ 110025, India \\ Tell: +91-9717200103 \\ Email: vivek2015@gmail.com
}

\begin{abstract}
Communication between mobile nodes is the key purpose of Mobile Ad Hoc Networks (MANETs). However, increase in the traffic leads to multiple challenging issues like congestion among nodes, consumption of more bandwidth, high energy requirement and low efficiency output. A powerful solution to these issues of the MANET is to use intelligent routing protocols. The Adaptive Neural Fuzzy Inference System (ANFIS) is one of those advanced technique which adjust itself according to the change in system. Therefore, the use of ANFIS in routing protocol results in efficient guiding of packet from source to destination. In this paper, the route selection mechanism of traditional Ad Hoc on Demand Distance Vector (AODV) routing protocol is improved with the use of ANFIS having input attributes hop count, energy consumption and delay. Simulation of the proposed ANFIS Aided AODV (AAODV) shows the clear improvement in quantitative parameters like Throughput and Packet Delivery Ratio (PDR).
\end{abstract}

Keywords: ANFIS, AODV, MANET, Intelligent Routing System

\section{Introduction}

Mobile ad hoc networks have the characteristics of less infrastructure, mobility, dynamic topology and the ability to communicate with each other even though they are not directly in the range. Due to these characteristics, MANETs have gained tremendous attention of researchers from the last decade. With the increase in mobile users, the demand of wireless applications has also significantly increased. To meet these requirements, canon of routing protocols have been already developed (Murthy and Manoj, 2004; Sharma and Alam, 2012). Based on table update mechanism, the previously reported routing protocols are divided into three groups (Murthy and Manoj, 2004; Pei et al., 2000; De Rango, 2010; Sharma and Alam, 2012): (1) ondemand; (2) table-driven; and (3) hybrid. On demand routing protocols share routing information whenever it is needed by nodes, while the table-driven routing protocols share the routing information periodically between nodes. The hybrid protocols are the combination of on demand as well as table driven protocols.

The MANETs are widely deployed in real time applications like military, conference room meeting, disaster management and multimedia etc. Routing plays a vital role in communication among data packets of
MANET. In case of multiple applications, the ad hoc networks are highly dependent on its environment variables like network topology, traffic strength, energy consumption, delay, scalability, security and bandwidth. While designing the protocol for applications of MANET, the above said environment variables should be considered for routing decision. To improve the routing decision, the effects of incorporating ANFIS to traditional AODV is considered. The ANFIS is highly capable of thinking, reasoning, decision making and have high degree of parallelism (Jang, 1993). The traditional AODV routing protocol consider only hop count as a metric in routing decision whereas the proposed ANFIS aided AODV (AAODV) consider three metrices hop count, nodeenergy and delay in routing decision.

The rest of the paper is organized as follows. In Section 2, the related works relevant to the areas of routing decision strategies is discussed, Section 3 discusses about the adaptive neural fuzzy inference system, Section 4 presents system model, Section 5 shows simulation results andSection 6 concludes the work.

\section{Related Work}

The aim of the routing protocol in MANET is to discover route in the network from source node to 
destination nodels so that the quality of service is enhanced. While designing routing protocol, one should consider challenges in MANETs like large delay, less energy, low trust factor, to find an optimal route among nodes. Delay of data packets between the nodes decrease the performance of network, if delay is small it is acceptable in most of the applications (Sheng et al., 2006; Wang et al., 2009; Zuo et al., 2010) but if delay is large significant information is lost. Sometimes, small delay also causes havoc like in real time multimedia applications. Packet lost due to congestion or death of certain node in MANET also degrade the network performance. So, the researchers also consider the average life time and trust factor while designing the routing protocol. Su et al. (2008) proposed modification of AODV routing protocol with fuzzy logic to multicast in mobile ad hoc network. The fuzzy logic based multi criteria of the AODV routing protocol is dynamically evaluated to determine the appropriate route and active route lifetime. Paul et al. (1999) proposed routing algorithm in which the route life time must be greater than some threshold value. Liang (2002) proposes the fuzzy logic system based routing protocol that dynamically calculate the route expiry time. Jiang et al. (2005) proposes routing protocol that uses the probability concept, Farkas et al. (2008) and Singhal and Daniel (2014) uses fuzzy based uses pattern matching approach to evaluate the node stability that increases the trust among nodes. Mallapur and Patil (2014) proposed fuzzy logic system based routing protocol for MANET that choose high quality path among the nodes. Pasupuleti et al. (2002; Song and Fang, 2006; Sheng et al., 2006), proposed adaptive routing algorithm based on fuzzy theory which aims to solve traffic management problem and achieve good trade-off between quality of service (QoS) and network performance. Since MANET is self-configured and completely mobile in nature therefore security becomes essential parameter while considering applications like military, banking. Therefore, many routing protocols are designed with security features along-with QoS. Nie et al. (2006; Jin et al., 2006) presented fuzzy logic and security based routing protocol that determine the most secure path for routing. In real time applications like rescue operations, the overhead to establish the route should be low, because the mobile node of MANET totally depends on its battery for power. Hence, battery life is very important and keeping this in mind researchers design energy aware routing algorithms. Torshiz et al. (2008) proposed modification in AODV protocol based on energy that provides the optimal route based on bandwidth and hop count of each route. To obtain the optimal route the authors, also incorporated fuzzy logic (Ross, 1995) to AODV for energy aware routing. Wang et al. (2005) proposed fuzzy based dynamic routing protocol that depends on degree of membership for configuring and categorizing the network. Ortiz et al. (2011) proposed the use of fuzzy logic based on energy aware metric to evaluate node conditions in AODV protocol, that selectthose nodes which are in better state for routing. Dehyadegari et al. (2011) proposed an adaptive routing algorithm based on fuzzy logic in which each link cost is dynamically determined for current network condition. Sharma et al. (2015). The researchers have provided many successful protocols in the recent past but however they are failed to incorporate adaptive learning strategies. Therefore, the model of proposed protocol presented in next section will include the advanced adaptive learning method ANFIS to the AODV routing protocol.

\section{Model Formulation}

In this section, we propose an Adaptive neural fuzzy inference aided system that improves the routing decision of AODV routing protocol. It stores the values and judge the preference according to design behavior of the system.

\section{Route Selection Metrics}

In the route path selection decision of AODV routing protocol (Perkins, 1999; Murthy and Manoj, 2004; Khimsara et al., 2009; Fahad and Ali, 2010) based on only hop count metric. The route path is also influenced by other variable like delay, energy, congestion etc. The Table 1 shows the major characteristic of on demand routing protocol when mobility is high and thus affect the routing decision. However only three variables will be considered in this model, consist of:

Hop count: Number of hopes between the sources to destination Residual energy (Ei) (Su et al., 2008): The remaining residual energy of a node is computed as multiplication of residual time $\mathrm{Ti}$ for a node in which its energy is consumed by a factor $E i$ with power energy consumption rate $P C R_{i}$ :

$E_{i}=T_{i} \times P C R_{i}$

where, $P C R_{i}$ is computed as:

$P C R_{i}=\frac{\left(P_{r} \times N_{r}+P_{s} \times N_{s}+P_{o} \times N_{o}\right)}{T}$

where, $P_{r}, P_{s}$ and $P_{o}$ is the power consumed when the network receives, sends and overhears of the packet respectively and $N_{r}, N_{s}, N_{o}$ is the total number of packets receives, send and overhears by a network respectively. 
Table 1. Characteristic of on demand routing protocols when mobility is high

\begin{tabular}{|c|c|}
\hline Characteristic & $\begin{array}{l}\text { On-demand routing } \\
\text { protocol }\end{array}$ \\
\hline Scalability & weak \\
\hline Adaptability of topology changes & weak \\
\hline Bandwidth & high \\
\hline Delay & large \\
\hline Power consumption & high \\
\hline Routing overhead & high \\
\hline
\end{tabular}

Delay: The delay is defined the difference of the packet arrival time $\left(P_{a r r}\right)$ for which its packet is arrived at node and acknowledged time $\left(P_{a c k}\right)$ at which data packet is acknowledged at a node. The average link delay for ' $m$ ' packet within time period $\left(T_{d}\right)$ is given as:

$$
D=\frac{\sum_{i=1}^{m}\left(P_{a c k}-P_{a r r}\right)}{T_{d}}
$$

Anf is cost value: It is the value computed at each node. The ith an fis cost is calculated by ANFIS system. These inputs are taken into account for types of variation in data values. Membership function and Rules depends on many parameters. If there is variation in these parameters then the different rule is applied to these variables. Based upon input parameter value each node is applied to ANFIS system and it provides the output value as cost. Based upon that value each node takes its routing decision.

\section{Adaptive Neural Fuzzy Inference System (ANFIS)}

ANFIS is a multi-layer adaptive network-based fuzzy inference system introduced by Jang (1993). The architecture of an ANFIS used in this paper consists of five layers, which is shown in Fig. 1. A hybrid learning mode is used to implement the different node functions to learn and tune parameters in a FIS. The ANFIS is an extension of the Takagi-Sugeno-Kang (TSK) fuzzy model (Ross, 1995; Rea and Pesch, 2004; Jin et al., 2006; Nie et al., 2006; Lima et al., 2008; Zuo et al., 2010) TSK or sugeno model is used in this paper that has advantage over high dimension problem.

\section{Layer 1. Generate the Membership Grade}

Every node $i$ in this layer with function is where $x$ is the input to node $i . A i, B i, C i$ is the linguistic label associated with this node function. We choose $\mu A(x)$ maximum equal to 1 and minimum equal to 0 :

$$
\begin{aligned}
& O_{1 i}=\mu_{A i}(x) \\
& O_{1 i}=\mu_{B i}(y) \\
& O_{1 i}=\mu_{C i}(z)
\end{aligned}
$$

where, $O_{1 i}$ is output of the node $i$ in a layer 1 .

\section{Layer 2. Generate the Firing Rule}

Nodes in this layer are labeled as $\pi$. Multiply the signal before outputting. The Output are given by:

$O_{2 i}=w_{i}=\mu_{A i}(x) \mu_{B i}(y) \mu_{c i}(z)$

\section{Layer 3. Normalize the Firing Strength}

Every node in this layer is fixed and labeled as $\mathrm{N}$ and Perform a normalization of the firing strength from the previous layer. The output of each node is given by:

$O_{3 i}=\bar{w}_{i}=\frac{w_{i}}{w_{1}+w_{2}+w_{3}}$

\section{Layer 4. Calculate rule Outputs based on the Consequent Parameters}

Output is the product of normalized firing rule and the order of first order polynomial and is given by:

$O_{4 i}=\bar{w}_{i} f i=w_{i}\left(p_{i} x+q_{i} y+r_{i}\right)$

where, $\left(p_{i}, q_{i}, r_{i}\right)$ are consequent parameters that deals with then part of fuzzy rule and it can be modified.

\section{Layer 5}

Sum of all the inputs from layer 4 . It compute the overall incoming signal:

$O_{5 i}=f=\sum_{i} \bar{w}_{i} f i=\frac{\sum_{i} w i f i}{\sum_{i} w i}$

ANFIS modifies the membership functions to the input/output data in order to account for types of variations in the data values, rather than choosing the parameter associated with a given membership function arbitrarily. Where the membership functions and rules depend on various parameters, changes these parameters will change the membership function rule which are saved in the knowledge base. Instead of looking the data to choose the membership function parameter or set the rule base, they can be modified automatically based on route choice logic through ANFIS.

\section{Path Selection in AODV}

In AODV (Perkins et al., 1999; Ko and Vaidya, 1998; Misra and Manda, 2005; Bai and Singhal, 2006; Manickam and Shanmugavel, 2007; Klein, 2008; De Rango et al., 2003), when a node wants to communicate with other node, in order to find a path, it broadcasts a RREQ packet to the network. RREQ packet contains the information of traversed nodes. Each node updates its routing table based upon the information stored in control message. 


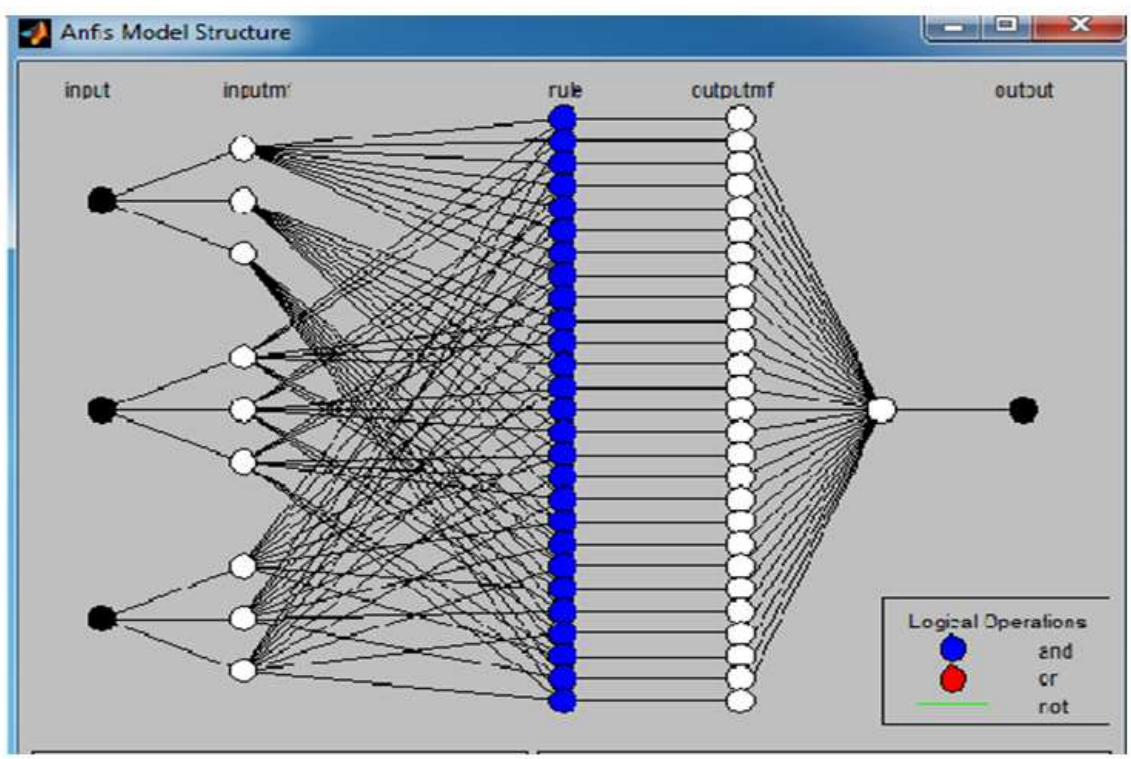

Fig. 1. Architecture of an ANFIS with 3 inputs (hop count, delay and energy) one output

When a node receives RREQ packet, it updates the route to source node. Afterwards it checks intermediate nodes accumulated in the path. A new entry is made in the routing table for any of the intermediate nodes, if one did not already exist. If a route entry for a node exists and if the hop count to any of the intermediate node is less than the previously known hop count to that node, the routing table entry is updated for that node. The entry is updated by retaining the previously known sequence number of that node.

\section{Path Selection in ANFIS based AODV}

Here, we propose a routing decision method to decide whether a node will appear in the path to continue or not. Route metrics that make the routing decision are energy of participating node, hop count and end-to-end delay between nodes. Three linguistic variables for inputs are: Low, Medium and High. Five linguistic variables for outputs are very low, low, medium, high and very high, where each one is assigned a value between $\{0,1\}$. Triangle membership's function is used to represent the output and input. These evaluations are passed through a fuzzy inference engine that applies a set of fuzzy rules to obtain the desired behavior of the system as shown in Table 2.

Due to the broadcast nature of route discovery process of AODV, the RREQ packet of AODV carries the fuzzy input parameter: hop count, delay, energy. Each node embedded a fuzzy logic system that dynamically evaluates the fuzzy cost each time when RREQ packet arrives. If it finds path with lesser fuzzy cost then it is chosen and update reverse route entry. This process is continued until get the destination. This scheme eradicates unsuitable paths from the route discovery process and optimizes the routing protocol.
Table 2. Rule base for ANFIS AODV

\begin{tabular}{lllll}
\hline Rule & Hop count & Energy & Delay & Output \\
\hline Rule1: & Low & Low & Low & Very High \\
Rule2: & Low & Medium & Low & Very Low \\
Rule3: & Low & High & Low & Very Low \\
Rule4: & Low & Low & Medium & Very High \\
Rule5: & Low & Medium & Medium & Low \\
Rule6: & Low & High & Medium & Low \\
Rule7: & Low & Low & High & Very High \\
Rule8: & Low & Medium & High & Medium \\
Rule9: & Low & High & High & Medium \\
Rule10: & Medium & Low & Medium & High \\
Rule11: & Medium & Medium & Medium & Low \\
Rule12: & Medium & High & Medium & Low \\
Rule13: & Medium & Low & Medium & High \\
Rule14: & Medium & Medium & Medium & Medium \\
Rule15: & Medium & High & Medium & Medium \\
Rule16: & Medium & Low & High & Very High \\
Rule17: & Medium & Medium & High & High \\
Rule18: & Medium & High & High & High \\
Rule19: & High & Low & Low & Medium \\
Rule20: & High & Medium & Low & Medium \\
Rule21: & High & High & Low & Medium \\
Rule22: & High & Low & Medium & Medium \\
Rule23: & High & Medium & Medium & High \\
Rule24: & High & High & Medium & High \\
Rule25: & High & Low & High & High \\
Rule26: & High & Medium & High & Very high \\
Rule27: & High & High & High & Very high \\
\hline & & & &
\end{tabular}

Unstable path is classified as paths that have a large associated signal loss, consists of low-energy nodes, high number of hops or paths spread over a large distance between source and destination.

The proposed algorithm is depicted in the given flowchart (Fig.2): 


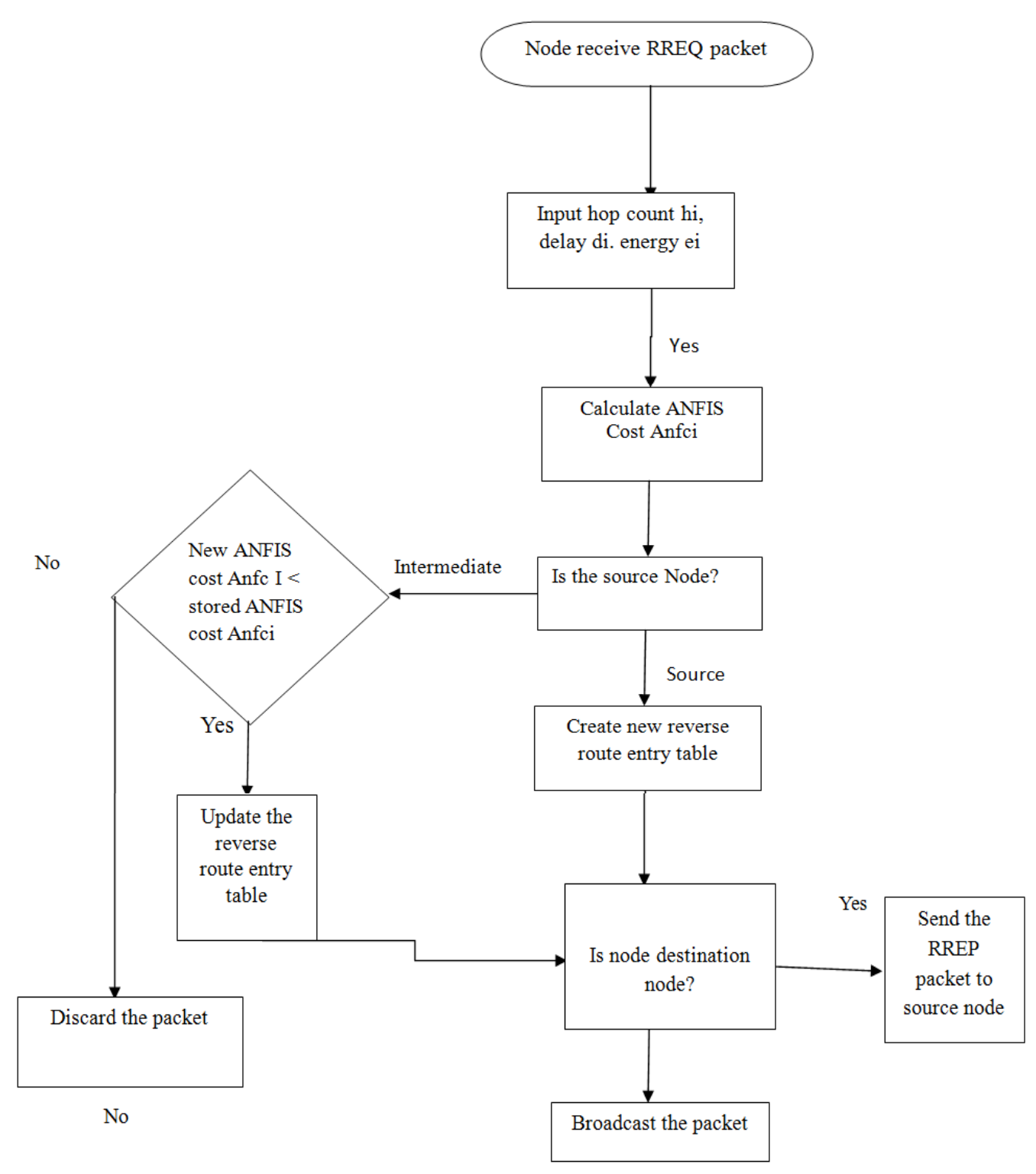

Fig. 2. Flowchart of proposed Algorithm (AAODV)

\section{Model Implementation}

The distance between two nodes is counted as one hop unit. The delay is the time taken by a packet to reach from one node to another. It ranges between 0 to $10 \mathrm{~ms}$.Energy is the total amount of energy available on each node, so that it can participate in routing process. Each node has its energy ranging from 0 to $100 \mathrm{j}$. To explain the above discussed terms, a hypothetical network consisting 14 nodes and 19 links is shown in Fig. 3. It can be seen that in the presented network, there are 6 feasible routes (a-g-l-m-n-k, a-b-c-d-e-f-k, a-b-c-dj-n-k ,a-b-c-I-j-n-k , a-b-h-I-j-n-k , a-g-h-I-j-n-k) from source node ' $a$ ' to destination node ' $k$ '.

\section{System Procedure}

Least cost path is chosen based on total cost calculated from three data inputs (i.e., hop count, energy, delay) listed in Table 3. Route a-g-l-m-n-k is recommended by the traditional AODV routing algorithm because it has the least hop count but the system decides to take route $a-b-h-i-j-n-k$ due to minimum total cost. Therefore, the system will recommend route a-b-h-i-j-n-k in the future. To perform the learning process, the costs interchanging between different routes will be the input for ANFIS data training. The total cost of various routes after the execution of training process are listed in Table 4. 


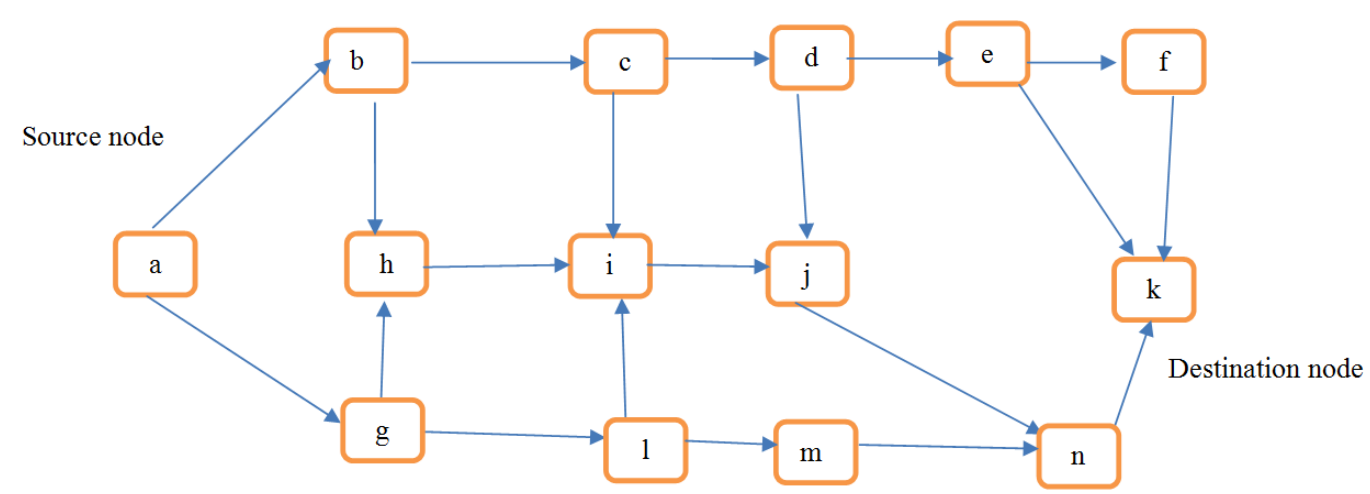

Fig. 3. Hypothetical network

Table 3. Input value of route affecting variable

\begin{tabular}{llllll}
\hline No & Link & $\begin{array}{l}\text { Hop count } \\
\text { (Normalized) }\end{array}$ & $\begin{array}{l}\text { Delay } \\
\text { (Normalized) }\end{array}$ & $\begin{array}{c}\text { Energy } \\
\text { (Normalized) }\end{array}$ & $\begin{array}{c}\text { Cost after } \\
\text { tuning }\end{array}$ \\
\hline 1 & $(\mathrm{a}, \mathrm{b})$ & 0.8000 & 0.289 & 1.000 & 0.6280 \\
2 & $(\mathrm{a}, \mathrm{g})$ & 0.6000 & 0.5879 & 0.700 & 0.6169 \\
3 & $(\mathrm{~b}, \mathrm{c})$ & 0.7000 & 0.379 & 1.000 & 0.6115 \\
4 & $(\mathrm{~b}, \mathrm{~h})$ & 0.8000 & 0.280 & 1.000 & 0.6082 \\
5 & $(\mathrm{c}, \mathrm{d})$ & 0.5000 & 0.620 & 0.900 & 0.6013 \\
6 & $(\mathrm{c}, \mathrm{I})$ & 0.4530 & 0.655 & 0.950 & 0.5934 \\
7 & $(\mathrm{~d}, \mathrm{e})$ & 0.6000 & 0.609 & 0.940 & 0.5868 \\
8 & $(\mathrm{~d}, \mathrm{j})$ & 0.4500 & 0.485 & 0.700 & 0.5835 \\
9 & $(\mathrm{e}, \mathrm{f})$ & 0.4400 & 0.510 & 0.800 & 0.5727 \\
10 & $(\mathrm{e}, \mathrm{k})$ & 0.4190 & 0.809 & 1.000 & 0.7402 \\
11 & $(\mathrm{f}, \mathrm{k})$ & 0.6190 & 0.599 & 1.000 & 0.5627 \\
12 & $(\mathrm{~g}, \mathrm{~h})$ & 0.5000 & 0.620 & 0.900 & 0.5171 \\
13 & $(\mathrm{~g}, \mathrm{l})$ & 0.6000 & 0.610 & 0.800 & 0.5864 \\
14 & $(\mathrm{~h}, \mathrm{I})$ & 0.4390 & 0.431 & 0.800 & 0.4706 \\
15 & $(\mathrm{I}, \mathrm{j})$ & 0.4390 & 0.435 & 1.000 & 0.4762 \\
16 & $(\mathrm{j}, \mathrm{m})$ & 0.6000 & 0.485 & 1.000 & 0.5011 \\
17 & $(\mathrm{n}, \mathrm{k})$ & 0.2610 & 0.800 & 0.700 & 0.5596 \\
18 & $(\mathrm{l}, \mathrm{m})$ & 0.5000 & 0.509 & 0.500 & 0.6131 \\
19 & $(\mathrm{~m}, \mathrm{n})$ & 0.9500 & 0.789 & & 0.7250 \\
\hline
\end{tabular}

Table 4. Route selection

\begin{tabular}{llll}
\hline Route Path & Cost before training & Cost after training 1 & Status \\
\hline a-b-c-d-e-f-k & 3.5630 & 3.5630 & \\
a-b-c-d-j-n-k & 3.4850 & 3.3958 & Least cost path after trg1 \\
a-b-c-I-j-n-k & 3.3698 & 3.0046 & Least cost path before trg. \\
a-b-h-I-j-n-k & 3.2437 & 3.0473 & 3.0063 \\
a-g-h-I-j-n-k & 3.1415 & & \\
\hline
\end{tabular}

It is observed that after the training, the total cost of route ab-c-i-j-n-k is minimum instead of a-b-h-i-j-n-k. Therefore, the system re-learns and finally recommends the new route.

\section{System Results}

The results have demonstrated some important characteristic of ANFIS based AODV routing protocol. Cost of route chosen by AODV detail is shown in Table 3. The system is designed to minimize the decision error close to zero. It means, the total cost of route recommended will match the cost of route chosen by system when decision error comes to zero.

\section{Simulation Model}

The environment variablesconsidered for simulation of model for proposed protocol are listed in Table 5. In an area of $700 \times 700 \mathrm{~m}^{2}, 50$ mobile nodes are placed. The channel capacity is fixed at $54 \mathrm{Mbps}$. A traffic generator to generate the CBR (Constant Bit Rate) traffic and the random waypoint mobility model are used. The speed of individual node ischoosen in the range of 0 to $10 \mathrm{~m} / \mathrm{s}$. The data payload size is set 512 bytes. These network parameters are simulated for 250 s by NS 2.35 simulator by using the system implemented with $\mathrm{C}++$ and $\mathrm{Tcl}$ language. ANFIS is implemented in MATLAB. 
Table 5. Simulation environment variables

\begin{tabular}{ll}
\hline Parameter & Values \\
\hline Routing Protocol & AODV, MBCR, FAODV, AAODV \\
No. of Nodes & 50 \\
Area & $700 \times 700 \mathrm{~m}^{2}$ \\
Channel Capacity & $54 \mathrm{Mbps}$ \\
Traffic type & CBR \\
Mobility Model & Random Way point \\
Simulation Time & $250 \mathrm{~s}$ \\
\hline
\end{tabular}

During the route discovery phase, ANFIS possessed with three inputs hop count, delay and energy. The fuzzifier rule base of ANFIS contains 27 rules and its defuzzifier has one output. To achieve highly stable and balanced route, data training sets are required. The data set comprising 1000 data points, which is divided into two sets of 300 and 700 data points for training and testing respectively. The first set is used to train the network and the model is validated with all the 1000 points including the 700 points that were not used in the training process. Data sets are derived from fuzzy 'if then rules'. The training data set is fed as an input to ANFIS tool under MATLAB environment. As a result, the optimal fuzzy membership function is obtained as an output which is used in NS2 environment to take the routing decision. The training is done for error tolerance 1e-5, epochs 100 and hybrid learning method.

To evaluate the proposed ANFIS based AAODV routing protocol, the following performance based metrics were used for simulation model:

- $\quad$ Packet Delivery Ratio (PDR): It can be expressed as the ratio of summation of all the packets that arrived at the destination nodes to the summation of all packets that are transmitted by the source nodes

- Average routing overhead load: It can be expressed as the ratio of summation of all the overhead routing control packets sent from all nodes within the entire MANET network over the simulation time

- Average network throughput: It can be expressed as the ratio of summation of data packets successfully arrived at the destination per unit of the simulation period time

The parameters considered for route selection method for the traditional AODV routing protocol, Minimum Battery Cost routing (MBCR) protocol 1(et al.), fuzzy AODV (FAODV) routing protocol and proposed AAODV protocol are presented in Table 6 . These parameters are simulated for varying pause time from 0 to $100 \mathrm{sec}$.

The performance comparison of all the above said protocols are presented in Fig. 4-6. The average network throughput of the proposed AAODV protocol is highest among the all and is observed in Fig. 4. The throughput is high due to less number of broken links are present in the network because higher the broken links leads to lower the successful number of transmitted packets at destination node.

\begin{tabular}{llll}
\multicolumn{2}{l}{ Table 6. Parameters considered for route selection method } \\
\hline & $\begin{array}{l}\text { Parameters considered } \\
\text { for Route Selection Method }\end{array}$ \\
& - Hop count & Delay & Energy \\
Protocol & $\sqrt{ }$ & $\times$ & $\times$ \\
\hline Traditional AODV & $\times$ & $\times$ & $\sqrt{ }$ \\
MBCR (C.K.Toh, 2001) & $\sqrt{ }$ & $\sqrt{ }$ & $\times$ \\
FAODV (Fahad,2010) & $\sqrt{ }$ & $\sqrt{ }$ & $\sqrt{ }$ \\
ANFIS aided AODV (proposed) & $\sqrt{ }$
\end{tabular}

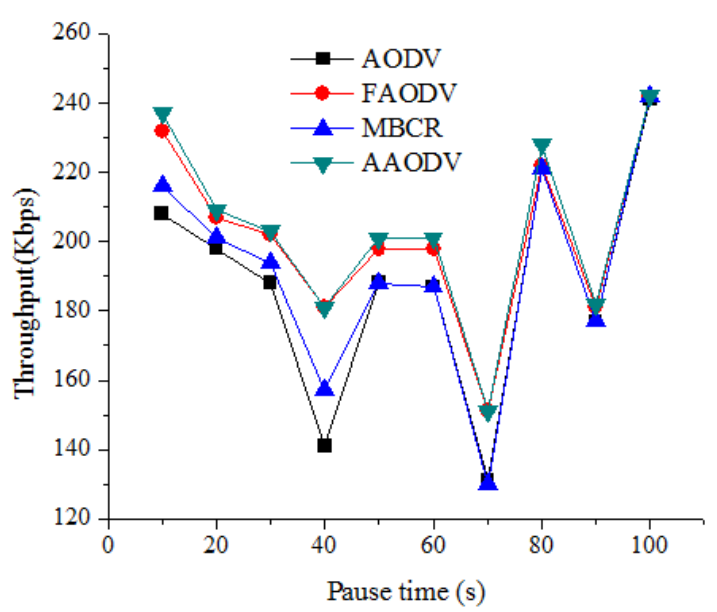

Fig. 4. Average Throughput Vs Pause Time

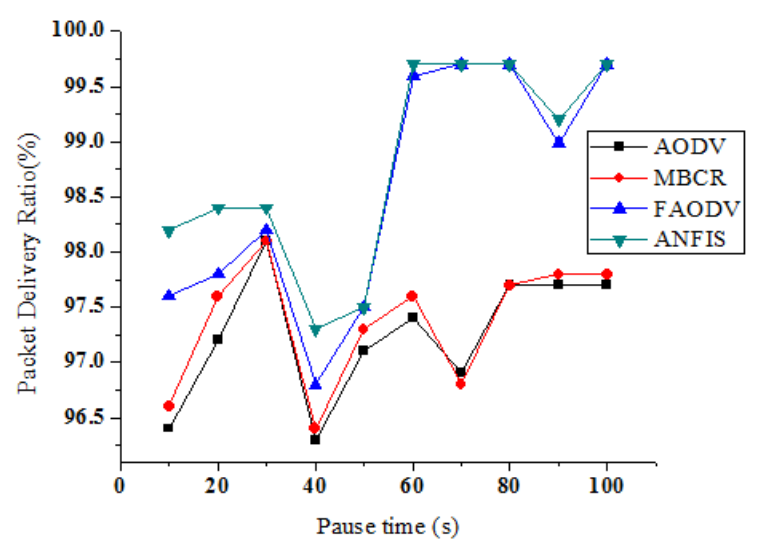

Fig. 5. Packet delivery ratio Vs pause time

The variations of packet ratio with respect to varying pause time for all these protocols are demonstrated in Fig. 5. The proposed AAODV protocol has the highest packet delivery ratio. This is due to consideration of delay and energy parameters in route selection of the proposed protocol.

The variations of routing load with respect to varying pause time for all these protocols are presented in Fig. 6. 


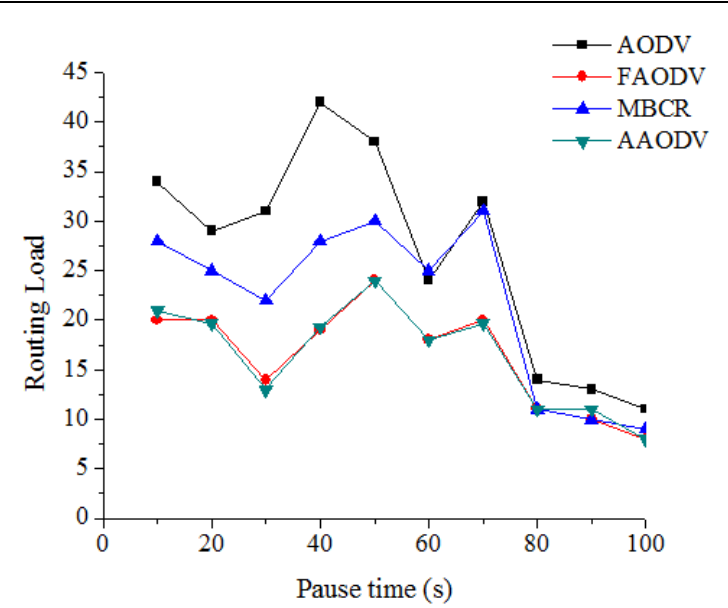

Fig. 6. Routing Load Vs Pause Time

From figure, it is observed that the proposed protocol has least route overhead (routing load) due to less number of broken links, low delay and due to contribution of nodes with high energy.

The proposed AAODV protocol is capable of predicting the optimal route and effectiveness of deploying the well trained ANFIS model in the AODV. In general, the proposed protocol AAODV has the highest performance among the state of art protocols.

\section{Conclusion}

In the study, new routing strategy that modifies AODV routing protocol in MANETis proposed. In AODV routing protocol the routing decision is based only upon hop count metric, however in real time scenario there are various parameters that affect the routing decision. Therefore, to find the optimal route, ANFIS system that deals with uncertain information is embedded into the AODV routing protocol. The proposed AAODV routing protocol has the inputs of hop count, delay and energy for routing decision. The comparison results of the proposed AAODV protocol shows the improvement in terms of throughput, PDR and routing load. The proposed AAODV protocol also have the potential to provide one direct path link from source node to destination node with higher efficiency and accuracy.

\section{Acknowledgement}

The authors are highly indebited to faculty and staff members of network laboratory at jamia milia islamia, new delhi to provide facilities for smooth conduct of this research work.

\section{Author's Contributions}

All the authors are equally responsible in this research work for collecting all the required data,simulation, analysis of results and manuscript prepartion.

\section{Ethics}

This publication is extended from an article named" Performance enhancement of AODV routing protocol using Adaptive neural fuzzy inference system" Vivek Sharma, Bashir Alam, M.N Doja. published in 7 th international Conference on Quality,Reliability,Infocom Technology and Business operation ,2015 .

\section{References}

Bai, R. and M. Singhal, 2006. DOA: DSR over AODV routing for Mobile Ad Hoc Networks. IEEE Trans. Mobile Comput., 5: 1403-1416. DOI: 10.1109/TMC.2006.150

De Rango, F., 2010. A modified location-aided routing Cprotocol for the reduction of control overhead in ad hoc wireless networks. Proceedings of the 10th International Conference on Telecommunication, (ICT'10).

De Rango, F., A. Iera, A. Molinaro and S. Marano, 2003. A modified location-aided routing protocol for the reduction of control overhead in ad-hoc wireless networks. Proceedings of the 10th International Conference on Telecommunications, Feb. 23-Mar. 1, IEEE Xplore Press, Papeete. DOI: 10.1109/ICTEL.2003.1191580

Dehyadegari, M., M. Daneshtalab, M. Ebrahimi, J. Plosila and S. Mohammadi, 2011. An adaptive fuzzy Logic-based routing algorithm for networks-onchip. Proceedings of the NASA/ESA conference on Adaptive Hardware and Systems, Jun. 6-9, IEEE Xplore Press, San Diego, pp: 208-214. DOI: 10.1109/AHS.2011.5963937

Fahad, T.O. and A.A. Ali, 2010. Improvement of AODV Routing on MANETs Using Fuzzy Systems. Proceedings of 1 st International Conference on Energy, Power and Control, Nov. 30-Dec. 2, IEEE Xplore Press, Basrah, Iraq.

Farkas, K., T. Hossmann, F. Legendre, B. Plattner and S.K. Das, 2008. Link quality prediction in mesh networks. Comput. Communi., 31: 1497-1512. DOI: 10.1016/j.comcom.2008.01.047

Jang, J.S.R., 1993. ANFIS: Adaptive-network-based fuzzy inference system. IEEE Trans. Syst. Man Cybernetics, 23: 665-685. DOI: 10.1109/21.256541

Jiang, S., D. He and J. Rao, 2005. A prediction-based link availability estimation for routing metrics in MANETs. IEEE/ACM Trans. Netw., 13: 1302-1312. DOI: 10.1109/TNET.2005.860094

Khimsara, S., K.K.R. Kambhatla, J., Hwang, S. Kumar and J.D. Matyjas, 2009. AM-AOMDV: Adaptive Multimetric Ad-Hoc On-Demand Multipath Distance Vector Routing. Proceedings of the International Conference on Ad Hoc Networks, (AHN'09), Springer International Publishing AG., pp: 884-895. 
Klein, A., 2008. Performance comparison and evaluation of AODV, OLSR and SBR in Mobile Ad-Hoc Networks. Proceedings of the 3rd International Symposium on Wireless Pervasive Computing, May, 7-9, IEEE Xplore Press, Santorini, Greece, pp: 571-575. DOI: 10.1109/ISWPC.2008.4556273

Ko, Y.B. and N.H. Vaidya, 1998. Location-Aided Routing (LAR) in mobile adhoc networks. Proceedings of the 4th Annual ACM/IEEE International Conference on Mobile Computing and Networking, Oct. 25-30, ACM New York, NY, USA, pp: 66-75. DOI: 10.1145/288235.288252

Liang, Q., 2002. Ad hoc wireless network Traffic-selfsimilarity and forecasting. IEEE Communi. Lett., 6: 297-299. DOI: 10.1109/LCOMM.2002.801327

Lima, M.N., H.W. da Silva, A.L. dos Santos and G. Pujolle, 2008. Survival Multipath routing for MANETs. Proceedigs of the IEEE Network Operations and Management Symposium, Apr. 7-11, IEEE Xplore Press, Salvador, pp: 425-432. DOI: 10.1109/NOMS.2008.4575164

Mallapur, S.V. and S.R. Patil, 2014. Fuzzy logic-based stable multipath routing protocol for mobile ad hoc networks. Porceedings of the Annual IEEE India Conference, Dec. 11-13, IEEE Xplore Press, Pune, India, pp: 23-24.

DOI: 10.1109/INDICON.2014.7030670

Manickam, J.M.L. and S. Shanmugavel, 2007. Fuzzy based trusted Ad Hoc On-demand distance vector routing protocol for MANET. Proceedings of the 3rd International Conference on Wireless and Mobile Computing, Networking and Communications, (CNC'07).

Misra, R. and C.R. Mandal, 2005. Performance comparison of AODV/DSR on-demand routing protocols for Ad Hoc networks in constrained situation. Proceedings of the IEEE International Conference on Personal Wireless Communications, Jan. 23-25, IEEE Xplore Press, New Delhi. DOI: 10.1109/ICPWC.2005.1431307

Murthy, C.S.R. and B.S. Manoj, 2004. Ad Hoc Wireless Networks: Architectures and Protocols. 1st Edn., Pearson Ltd.

Nie, J., J. Wen, J. Luo, X. He and Z. Zhou, 2006. An adaptive fuzzy logic based secure routing protocol in mobile ad hoc networks. Fuzzy Sets Syst., 157: 1704-1712. DOI: 10.1016/j.fss.2005.12.007

Ortiz, A.M., F. Royo,T. Olivares and L. OrozcoBarbosa, 2011. Intelligent route discovery for zigbee mesh networks. Proceedings of the IEEE International Symposium on World of Wireless, Mobile and Multimedia Networks, Jun. 20-24, lEEE Xplore Press, Lucca, pp: 1-6.

DOI: 10.1109/WoWMoM.2011.5986179
Pasupuleti, A., A.V. Mathew, N. Shenoy and S.A. Dianat, 2002. Fuzzy system for adaptive network routing. Proceedings of the 4th Digital Wireless Communications, (DWC'02), SPIE. Digital Library, Orlando, FL, United States.

DOI: $10.1117 / 12.472955$

Paul, K., S. Bandyopadhyay, A. Mukherjee and D. Saha, 1999. Communication-aware mobile hosts in ad-hoc wireless network. Proceedings of the IEEE International Conference on Personal Wireless Communication, Feb.17-19, IEEE Xplore Press, Jaipur, pp: 83-87.

DOI: 10.1109/ICPWC. 1999.759590

Pei, G., M. Gerla and T.W. Chen, 2000. Fisheye state routing: A routing scheme for ad hoc wireless networks. Porceedings of the IEEE International Conference on Communications, Jun. 18-22, IEEE Xplore press, New Orleans.

DOI: $10.1109 /$ ICC.2000.853066

Perkins, C.E. and E. Royer, 1999. Ad-hoc on-demand distance vector routing. Proceedings of the 2nd IEEE Workshop on Mobile Computing Systems and Applications, Feb. 25-26, IEEE Xplore Press, New Orleans. DOI: 10.1109/MCSA.1999.749281

Rea, S. and D. Pesch, 2004. Multi-metric routing decisions for ad hoc networks using fuzzy logic. Proceedings of the 1st International Symposium on Wireless Communication Systems, Sept. 20-22, IEEE Xplore Press, Mauritius, Mauritius, pp: 403-407. DOI: 10.1109/ISWCS.2004.1407278

Ross, T.J., 1995. Fuzzy Logic with Engineering Applications. 1st Edn., McGraw-Hill, New York, ISBN-10: 0070539170, pp; 600.

Sharma, V. and B. Alam, 2012. Unicast routing protocols in mobile ad hoc networks: A survey. Int. J. Comput. Appli., 51: 148-153.

Sharma, V., B. Alam, M.N. Doja, 2015. Fuzzy-based MAODV routing protocol in MANETs. Proceedings of the 2nd International Conference on Computer and Communication Technologies, (CCT'15), Springer International Publishing AG, pp:773-780.

Sheng, H.M., J.C. Wang, H.H. Huang and C.D. Yen, 2006. Fuzzy measure on vehicle routing problem of hospital materials. Expert Syst Applic., 30: 367-377. DOI: 10.1016/j.eswa.2005.07.028

Singhal, A. and A.K. Daniel, 2014. Fuzzy logic based stable on-demand multipath routing protocol for mobile ad hoc network. Porceedings of the 4th International Conference on Advanced Computing and Communication Technologies, Feb. 8-9, IEEE Xplore Press, Rohtak, pp: 23-30.

DOI: 10.1109/ACCT.2014.83

Song, W. and X. Fang, 2006. Multi-metric QoS routing based on fuzzy theory in wireless mesh network. Proceedings of the IET International Conference on Wireless, Mobile and Multimedia Networks, Nov.6-9, IEEE Xplore Press, Hangzhou, pp: 1-4. DOI: 10.1049/cp:20061184 
Su, B.L., M.S. Wang and Y.M. Huang, 2008. Fuzzy logic weighted Multi-criteria of dynamic route lifetime for reliable multicast routing in ad hoc networks. Expert Syst. Applic., 35: 476-484. DOI: $10.1016 /$ j.eswa.2007.07.010

Toh, C. K., 2001. Maximum battery life routing to support ubiquitous mobile computing in wireless ad hoc networks. IEEE Communications Magazine. DOI: $10.1109 / 35.925682$

Torshiz, M.N., H. Amintoosi and A. Movaghar, 2008. A fuzzy energy-based extension to AODV routing. Proceedings of the International Symposium on Telecommunications, Aug. 27-28, IEEE Xplore Press, Tehran, pp: 371-375.

DOI: 10.1109/ISTEL.2008.4651330

Wang, C., S. Chen, X. Yang and Y. Gao, 2005. Fuzzy logic-based dynamic routing management policies for mobile ad hoc networks. Proceedings of the Workshop on High Performance Switching and Routing, May 12-14, IEEE Xplore Press, Hong Kong, pp: 341-345.

DOI: 10.1109/HPSR.2005.1503251
Wang, X., Y.L. Yang and J.W. An, 2009. Multi-metric routing decisions in VANET. Porceedings of the IEEE International Conference on Dependable, Autonomic and Secure Computing, Dec. 12-14, IEEE Xplor Press, Chengdu, pp: 12-23. DOI: 10.1109/DASC.2009.37

Zuo, J., S.X. Ng and L. Hanzo, 2010. Fuzzy logic aided dynamic source routing in cross-layer operation assisted ad hoc networks. Porceedings of the IEEE 72nd Vehicular Technology Conference Fall, Sept. 6-9, IEEE Xplore Press, Ottawa, pp: 1-5. DOI: 10.1109/VETECF.2010.5594508 